\title{
Commémorer Deng et critiquer la " gauche »
}

Synthèse commentée

\section{Michal Meidan}

\section{CpenEdition}

\section{Journals}

Édition électronique

URL : http://journals.openedition.org/perspectiveschinoises/2883

ISSN : 1996-4609

\section{Éditeur}

Centre d'étude français sur la Chine contemporaine

\section{Édition imprimée}

Date de publication : 15 juillet 2007

ISSN : 1021-9013

\section{Référence électronique}

Michal Meidan, «Commémorer Deng et critiquer la « gauche » », Perspectives chinoises [En ligne], 2007/2 | 2007, mis en ligne le 03 avril 2008, consulté le 01 mai 2019. URL : http://

journals.openedition.org/perspectiveschinoises/2883 


\title{
Commémorer Deng et critiquer la "gauche "
}

\author{
Synthèse commentée de Michel Meidan d'après : \\ - Yuan Weishi ${ }^{(1)}$, Saisir les opportunités historiques ; se doter d'un courage et de l'ingénuité, promouvoir le processus de \\ réforme ", Nanfang dushi bao, 29 janvier 2007, page éditoriale.
}

- Article non attribué, " la Chine reste sur la voie de la réforme et de l'ouverture ", Xinhua, 19 février 2007.

$\mathrm{D}$ eux commémorations ont été mises en exergue en janvier et février 2007 : celle du décès de Deng Xiaoping intervenu le 19 février 1997, mais aussi sa célèbre " tournée vers le Sud ", qui avait relancé l'ouverture économique après les événements de Tian'anmen.

Dans un contexte politique mouvementé en préparation du $17^{\mathrm{e}}$ Congrès du PCC, à l'automne cette année, l'héritage politique et économique de Deng Xiaoping est ainsi invoqué par des partisans d'une poursuite vigoureuse des réformes, en réaction à certains " éléments de gauche " du gouvernement. $\mathrm{Hu}$ Jintao et Wen Jiabao se positionnent clairement en réformateurs « dengistes ». Une série d'articles parue dans la presse officielle replace le tandem dans la continuité d'un héritage libéral, à la fois économique et politique, tout en affichant une volonté de rectifier les problèmes, et principalement celui de l'inégalité croissante des revenus qui est la plus importante conséquence non voulue du programme de Deng Xiaoping. La presse officielle loue ainsi les acquis économiques des réformes de Deng, et aborde de façon plus circonspecte la réforme politique. Elle s'attache toutefois aussi à certains succès, notamment à la succession politique stable et réussie entre la troisième et la quatrième génération, à la promulgation de lois et de règlements nouveaux, et surtout, à la lutte contre la corruption.

Yuan Weishi va plus loin dans son analyse. Les louanges à Deng Xiaoping ne sont pas moindres que celles de l'agence de presse officielle, Xinhua, mais elles servent ici d'argument pour plaider contre un virage à gauche.

L'auteur se sert de l'héritage de Deng, avec maintes citations de ses travaux, afin de plaider la poursuite des réformes économiques, la participation à l'OMC et l'intégration de la Chine dans la mondialisation : " aucune solution économique ne nécessite l'abandon de cette voie ». Selon lui, la peur de la mondialisation et de la libéralisation accrue des marchés émane du sentiment que celles-ci puissent mettre en péril la sécurité nationale, ou conduire à une nouvelle colonisation de la Chine, cette fois-ci par le capital étranger. La réponse, plaide l'auteur, ne passe pas par des voies " non pacifiques », et de fait, la poursuite de l'intégration de la Chine dans les flux mondiaux rendrait ces craintes insignifiantes.

Le monde contemporain, poursuit-il dans son raisonnement, est bâti sur des fondations économiques et sur la maximisation des profits, mais la tradition de lutte [référence à la notion maoïste selon laquelle tout progrès émane d'un processus de lutte] et de sentiment victimaire de la Chine par des puissances impériales ne peut plus guider le pays. Certes, certains États interviennent dans la vie économique, mais ceci ne change pas le caractère fondamentalement commercial des interactions internationales.

Deuxièmement, certains en Chine sont incapables de se démarquer du modèle soviétique qui a " été une tragédie politique, économique, culturelle et idéologique ». Ce qui mène ces personnes à privilégier la planification économique au détriment des marchés, la gestion directe des entreprises et le monopole des marchés. De plus, "[Ces personnes] spolient le travail académique et [portent atteinte à] la liberté de pensée et de parole ".

L'auteur dénonce également l'intervention de la politique dans les choix économiques. La Chine aurait, selon lui, manqué trop d'opportunités historiques et il ne faudrait pas

1. Yuan Weishi est professeur d'histoire à l'université Sun Yatsen, à Guangzhou. II est l'auteur d'un article, paru le 24 janvier 2006 dans le magazine Freezing Point (Bingdian), un supplément du Quotidien de la Jeunesse, critiquant ouvertement le Parti communiste chinois (PCC) à propos de sa présentation de la guerre des Boxers dans les manuels d'histoire. Le magazine avait été fermé à la suite de cet article. 
en laisser passer d'autres, avertit-il, faisant écho ainsi aux propos du Premier ministre, Wen Jiabao ${ }^{(2)}$.

Mais le plus grand défi à relever reste, selon lui, celui du gouvernement par la loi. La corruption, un marché anarchique, les écarts de revenus et une faible capacité à l'innovation, telles sont les quatre grandes questions qui vont déterminer l'avenir de la Chine. Il appelle donc à la poursuite de la réforme constitutionnelle du pays. Les principes du gouvernement par la loi ont été intégrés à la Constitution du pays, et les dirigeants se sont exprimés concernant l'importance de cette réforme. Néanmoins, elle tarde à se réaliser même si les " citoyens " sont de plus en plus conscients de leurs droits et libertés ${ }^{(3)}$, sollicitant davantage le soutien d'avocats ou des médias. "Les organisations civiles se développent et donnent une voix aux victimes des injustices ", mais le système doit intégrer la protection de ces droits, faute de quoi, il reste impuissant.

L'éditorial de Yuan Weishi, un an après la fermeture de Bingdian, cible principalement les départements de la propa- gande, responsables de la fermeture du supplément du journal et de l'interdiction de la publication de huit ouvrages ${ }^{(4)}$ qui passent en revue des événements historiques et sociaux de l'histoire contemporaine de la Chine. La critique de l'auteur reste, prudemment, limitée aux thèmes de débats qui font la une de la presse chinoise de façon plus consensuelle. Ses propos interviennent toutefois dans un contexte de transformations politiques et de débats à l'intérieur de la Chine quant à la limite des réformes à suivre.

En dépit de cet éditorial critique et d'une tendance à revendiquer une libéralisation croissante, $\mathrm{Hu}$ Jintao semble poursuivre une ligne de réforme graduelle, et surtout consensuelle ${ }^{(5)}$. Les promotions à la tête des provinces et aux nouveaux postes ministériels traduisent l'équilibre auquel il souhaite arriver avec les autres factions. L'article de Yuan Weishi, tout en étant critique, emploie pour ce faire une terminologie politiquement correcte, mais reste une manifestation patente des débats qui occupent l'intelligentsia chinoise.

\section{Une politique active de "désinisation "}

\section{Synthèse commentée de Mathieu Duchâtel à partir de :}

- "La nouvelle version des manuels d'histoire nous permet de retrouver le vrai visage de l'histoire de Taïwan ", éditorial, Ziyou Shibao (Liberty Times), ler février 2007. Chang Chingwei, "La version modifiée des manuels d'histoire du lycée abandonne un mythe de manière neutre ", Xin Taïwan Xinwen Zhoukan, ${ }^{\circ}$ 567, ler février 2007.

- Wang Yifeng, "L'identité nationale, il faut en planter les graines à partir des manuels scolaires ", Xin Taïwan Xinwen Zhoukan, $n^{\circ} 567$, ler février 2007.

- "L'indépendantisme légaliste heurte un mur, l'indépendantisme psychologique s'enflamme ", éditorial, Lienhebao, 2 février 2007.

- Philip Yang Yungming, "Rectifier les noms à tout prix, c'est jouer sur la corde sensible ", Lienhebao, 11 février 2007, p. 15.

- Lin Huowang, "Chen Shuibian rectifie les noms pour sauver sa peau après 2008 ", Lienhebao, 12 février 2007, p. 15.

$\mathrm{D}$ epuis le début de l'année 2007, trois questions dominent la politique intérieure taiwanaise : les affaires de corruption, la sélection par les partis politiques de leur candidat aux présidentielles de 2008, et la campagne de désinisation (去中國化) orchestrée par les autorités de l'île. Cette dernière possède des dimensions stratégiques et idéologiques. Depuis la fondation du DPP, la désinisation représente l'une des revendications principales de l'aile la plus indépendantiste du parti. Pour ses défenseurs, elle représente l'aboutissement d'un effort légitime pour recentrer le régime de la République populaire de Chine sur Taïwan. Elle vise à corriger la sinisation forcée des Taiwanais par le Kuomintang et à libérer une identité nationale étouffée par un régime autoritaire attaché au mythe de la Grande Chine. Pour ses détracteurs, au contraire, cet effort de désinisation s'appuie sur des calculs populistes et sur une idéologie indépendantiste fondamenta-

2. Voir « La Culture institutionnelle selon Wen Jiabao » dans ce même numéro

3. Voir l'ouvrage récent de Kevin 0'Brien et Lianjiang Li, Rightful resistance in rural China, Cambridge University Press, 2006.

4. "Eight books banned in crackdown on dissent", South China Morning Post, 19 janvier 2007

5. Willy Wo-Lap Lam, "Striving for Balance: Assessing Recent Municipal and Provincial Leadership Changes", China Brief, vol. 7, no. 2, 18 avril 2007 